\title{
IMAGE SEGMENTATION BASED ON Multiplex NeTwORKS AND SUPER PiXELS
}

\author{
Ivo S. M. de Oliveira ${ }^{1,2}$, Oscar A. C. Linares ${ }^{1}$, Ary H. M. de Oliveira ${ }^{3}$, \\ Glenda M. Botelho ${ }^{3}$ and João Batista Neto ${ }^{1}$ \\ ${ }^{1}$ Instituto de Ciências Matemáticas e de Computação, Universidade de São \\ Paulo, \\ Caixa Postal 668, 13560-970, São Carlos, Brazil \\ ${ }^{2}$ Instituto Federal do Tocantins, Campus de Paraíso do Tocantins, \\ Caixa Postal 151, 77600-000, Paraíso do Tocatins, Brazil. \\ ${ }^{3}$ Universidade Federal do Tocantins, Palmas, Brazil
}

\begin{abstract}
Despite the large number of techniques and applications in the field of image segmentation, it is still an open research field. A recent trend in image segmentation is the usage of graph theory. This work proposes an approach which combines community detection in multiplex networks, in which a layer represents a certain image feature, with super pixels. There are approaches for the segmentation of images of good quality that use a single feature or the combination of several features of the image forming a single graph for the detection of communities and the segmentation. However, with the use of multiplex networks it is possible to use more than one image feature without the need for mathematical operations that can lead to the loss of information of the image features during the generation of the graphs. Through the related experiments, presented in this work, it is possible to identify that such method can offer quality and robust segmentations.
\end{abstract}

\section{KEYWORDS}

community detection; complex networks; image segmentation; multiplex networks; super pixels

\section{INTRODUCTION}

Image segmentation is one of the most important techniques used in Digital Image Processing to extract information from an image. It allows to decompose the image into two or more parts (regions or objects) [1], and it allows to extract information that can be used in subsequent processes of computational vision. Among the various applications we can mention: diseases diagnoses, allowing the identification of anomalies and the measurement of tissues, recognition of individuals, identification of several objects and industrial analysis.

Despite the large number of existing techniques, digital image segmentation is still an open field, due to the subjectivity and complexity of subdividing of the image into the most diverse scenarios and purposes. Techniques of image segmentation can use a variety of strategies and features, or a combination of them to achieve the goal, which is a correct segmentation. 
Some common features in carrying out the segmentation are: intensity values of luminance and chrominance, textures and morphological forms. The known segmentation strategies come mainly from the methods of point, line and edge detection, thresholding, and region-based techniques [1]. The segmentation of images may be slow, depending on the computational resources, method and resolution used.

Several works, [2], [3], [4], [5], [6], [7], use graphs to represent the elements of an image to perform the segmentation process. Once the graphs can represent any discrete data, model relationships and use several functions with combinatorial optimization, making feasible the construction of widely efficient solutions [8]. An approach that has emerged in this context is the modelling of an image as a graph, for further segmentation through community detection algorithms, which generally use algorithms such as Efficient Label Propagation [9] [10] [11], Fast Greedy [12] [13] and Louvain Method [14]. Such an approach is directly related to the area of complex networks.

Through more detailed studies of the concepts of complex networks it is possible to identify a modality that has been explored to improve the analysis of the different types of networks, which are the networks of multiple layers. They have become promising because they allow the analysis of networks with multiple resolutions that vary over time, multiplex networks and hybrid networks with the characteristics presented [15] [16]. These networks allow to assign a greater number of relevant features to the context in the formation of the communities, seeking to offer greater precision in the various applications.

The studies of the multilayer networks are not recent, but they were stagnant and have now been resumed becoming one of the main research topics of the network science. Over the years, monoplex networks have been extensively explored and today researchers are seeking to extend and generalize their various features and functions for multilayer networks. However, such extensions and generalizations are not simple given that new phenomena arise given the new peculiarities.

The detection of communities in multilayer networks is already something more recent and does not yet have several concrete concepts, even the concept of degree that is somewhat trivial in single-layer networks, does not yet have a well-defined generalization in the multilayer structure. There are extremely rare researches that address segmentation of digital images in the context of multilayer networks or multiplex networks, reflecting the difficulty of generalizing certain trivial concepts in the area of complex networks.

Hu et al. in [7] present a proposal of image segmentation using community detection in multilayer networks based on the modularity measure presented by Mucha et al. in [15]. However, the authors have identified that the computational cost and the analysis and characterization of the performance of the modularity optimization for such application is still a challenge.

This work seeks to broaden the segmentation quality observed in previous research, extending the proposal for the use of multilayer networks of multiplex characteristics for the segmentation of images, in which each layer will use different features, being able to relate values of intensity of luminance, chrominance and simple characteristics of the regions of the images. Finally, an image segmentation method is presented, with pre-segmented images through super pixels, through the decomposition of the image into several features (luminance, chrominance and histograms), 
modelling it through algorithms of multiplex networks to images represented as graphs, with reasonable processing time and with high precision.

As a main contribution of this work it is possible to quote the organization of an approach that initially contemplates the use of several features of the images in specific networks to allow the generation of specific communities for the accomplishment of segmentation of images through the detection of communities. Where such an approach provides a performance that enables its application in large synthetic and real images.

This article is organized as follows: Related work is presented in Section 2. The methodology is presented in Section 3. Results and discussion are in Section 4. Finally, conclusions are addressed in Section 5.

\section{RELATED WORK}

Browet et al. [14] present a technique based on graphs to detect segments or contours of objects in a given image. The algorithm is based on an approximation of the Louvain method (community detection algorithm) that deploys the community structures in a large graph. However, the optimal definition of a contour requires adjustment of parameters depending on the user or the application. The obtained communities are hierarchical, allowing to find sub-regions within an object. Through the realized experiments it is possible to observe that the technique avoids over segmentation through a weighted modularity scheme. The technique has a good performance of time and quality, but initially it only acts in grayscale images and it has clear dependence of parameters for the diverse applications.

However, Linares et al. [17] presented a new approach to segmentation of high-resolution images through the use of community detection and super pixels, reaching, through this combination, precision and a low processing time in the segmentation. For the detection of communities, the Fast Greedy algorithm was used and for the generation of super pixels, the Speeded-up Turbo

Pixels. However, the graph used to generate community detection was obtained by sum of the Euclidean distance of the three CIELAB channels.

As presented in the introduction, $\mathrm{Hu}$ et al. in [7] present a technique based on multilayer networks for the segmentation of images. The algorithm of community detection has the modularity based on the generalization presented by Mucha et al. in [15] and is presented in equation (1), below:

$$
Q_{m u l t i}=\frac{1}{2 \mu} \sum_{i j s r}\left[\left(A_{i j s}-\gamma_{s} \frac{k_{i s} k_{j s}}{2 m_{s}}\right) \delta_{r s}+\delta_{i j} C_{j s r}\right] \delta\left(g_{i s}, g_{j r}\right),
$$

However, for the authors, an obstacle identified in the application of the technique was the computational performance, since each pixel was represented in the graph as a vertex.

\section{Methodology}

The diagram of Figure 1 generally illustrates the proposed method of segmentation through community detection algorithms in Multiplex Network and Super Pixels. Given an input image, 
the pre-processing is applied by super pixels, considering several different features (intensity, color and others). Each of these features, or the super pixels that represent them, will form a layer of the network. After the generation of multiple graphs, the vertices are connected between the graphs, forming a multiplex network. The final segmentation is obtained by means of a community detection algorithm on the multilayer network that represents the image

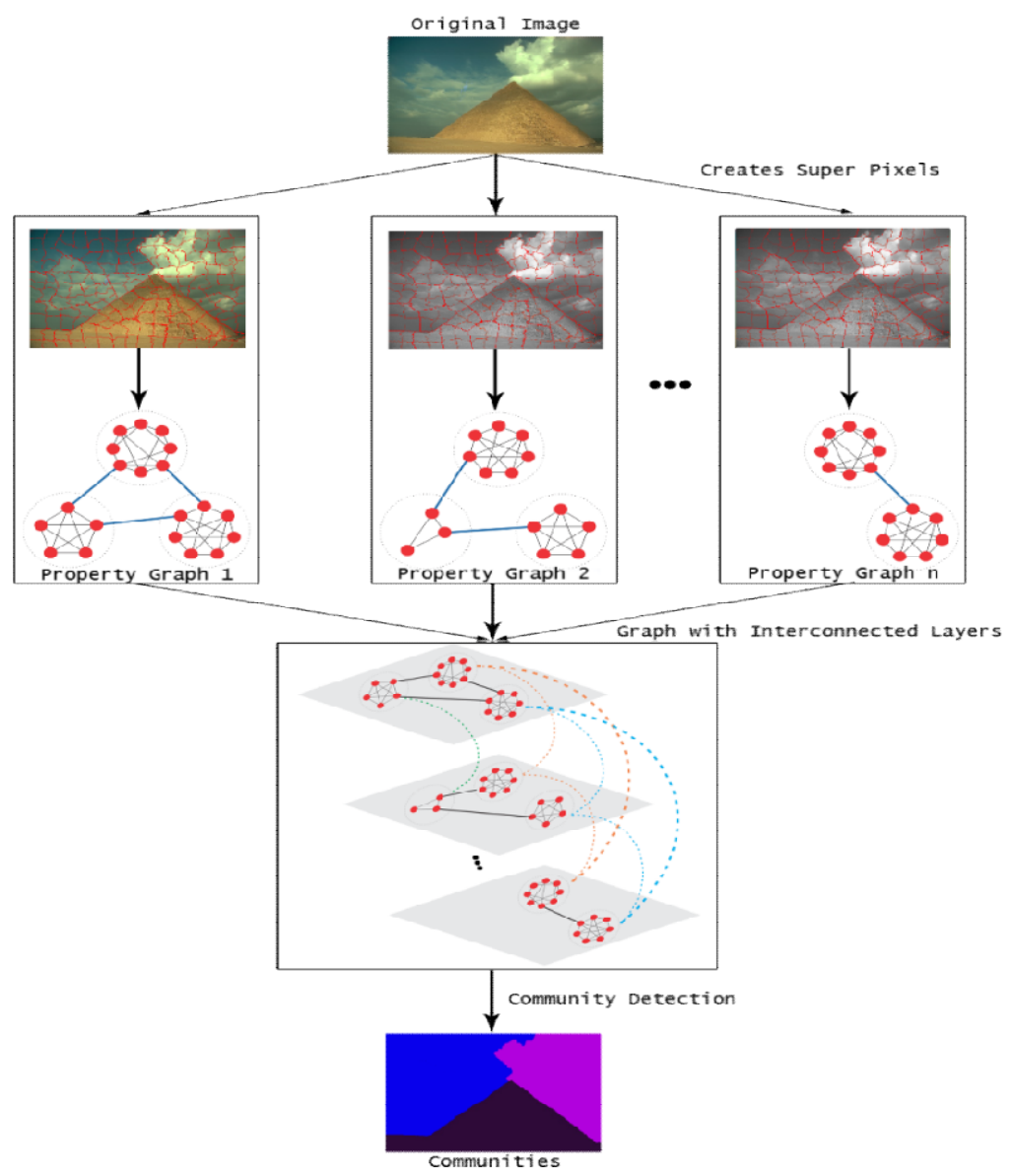

Figure 1. Proposed method of Image Segmentation by Means of Community Detection Algorithms in Multiplex Network and Super Pixels

\subsection{PRE-PROCESSING - CREATION OF SUPER PIXELS}

When receiving an original image this step consists of obtaining the super pixels of an image based on the various features of the image, in this step the main challenge is to obtain an algorithm extremely fast and effective in relation to the quality of pre-segmentation. The algorithm of generation of super pixels used was the Speeded-up Turbo Pixels [6], because it presents a good performance of time and quality in the pre-segmentation defined. The parameters used were the same ones observed in other studies [6] [17], among them: the size of super pixels, $10 \times 10$; number of iterations $=6$ and the parameters $\lambda 1=1$ and $\lambda 2=0.9$. 
However, it should be emphasized that the size of the super pixel influences the execution time of this stage and the quality of the segmentation, for images with different resolutions it is necessary to revise these parameters. Based on the super pixels, it is possible to generate the graphs that will compose the layers of the multilayer network, the methodology of connecting the vertices of such graphs is presented in the next section.

\subsection{GRAPH GENERATION}

In the creation of the multiple graphs it is essential to emphasize that it is composed of vertices obtained through the super pixels, which can be represented, for example, through the medium intensity, the average of each channel for the various LBP (Local Binary Pattern) color models and histograms for textures, among others. Already the edges are obtained by calculating the weight of a given property of two vertices of the graph. Therefore, if the weight between two vertices is less than a threshold $t$, a connection is established, where $t$ can be modified based on the similarities of the pixels.

For all features the weight function can be defined by equation (2):

$$
W_{i, j}=1-\left|I_{i}-I_{j}\right| \leq t
$$

in which, Ii offers for example the average intensity of the super pixel $\mathrm{i}$.

The threshold $t$ is relative to the characteristics of the images. When it has a very high value, such a threshold can generate communities with mixed image regions. When it has a very low value, such threshold can generate over-segmentation in the image. Therefore, an adaptive threshold will be useful and adequate, consisting of the following steps for each super pixel:

1. Initially, a low threshold is displayed; if the super pixel has no connection, go to step 2;

2. The threshold is incremented, if the super pixel has no connection, repeat step 2 until the vertex has at least one connection.

To avoid the connection of super pixels very far, a radius $=5$ (super pixels) will be applied, as observed in literature [17]. The Figure 2 illustrates the use of the radius in the proposed method. For each defined feature will be generated a graph with vertices based on their respective super pixels. Since a multiplex network is created, all the vertices of one layer will be connected with the corresponding vertices of the other layers. 


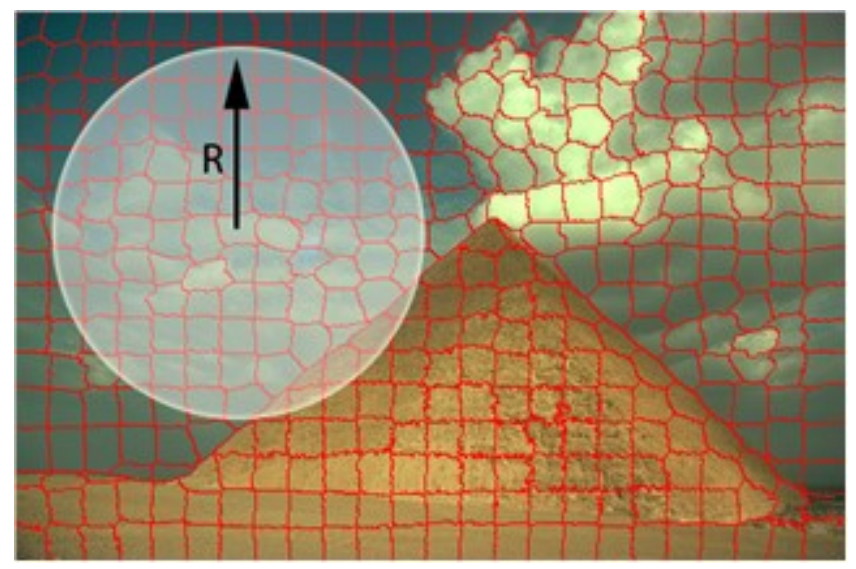

Figure 2. Pre-segmented image with super pixels illustrating a radius in which the super pixels will make connections in the creation of a graph

The creation of the graphs was implemented using the Igraph library in Language C. The process of image segmentation by community detection is presented in the next section and uses the graph for community detection.

\subsection{Image Segmentation By Detection Of Communities}

The proposed community detection process is based on the exploration of the most consolidated multilayer network algorithms in the literature and implemented in the MuxViz tool [18]. Among the algorithms used are the multiplex Infomap and the Louvain. The Louvain algorithms used adjacency sums to allow analysis of a multiplex network. Based on the communities generated by the MuxViz tool using the mentioned algorithms, the super pixels of the same community are united generating the segmentation of the image. These experiments aim to present the characteristics of the segmentation based on the proposed method exploring two algorithms of detection of different communities, Infomap and Louvain

\section{EXPERIMENTAL RESUltS AND COMPARISONS}

The results of two experiments for the analysis of the proposed method are presented below. All experiments were performed on an Intel Core i7 $2.9 \mathrm{GHz}$ computer with $4 \mathrm{~GB}$ RAM and the Ubuntu/Linux Desktop Operating Systems 64 bits 12.10 and Linux Mint 18.264 bits (Detection Communities).

\subsection{Experiment A: Segmentation Of Real Images With Infomap (Multiplex NeTWORKS) AND LOUVAin (MUltiplex Networks)}

In this experiment, image segmentation based on a real image randomly selected from the Berkeley image database is being compared. In order to observe the segmentation characteristics in each algorithm for real images. The time of realization of each algorithm is also observed. It is important to emphasize that the generation of the detection of the communities was using the MuxViz tool and the features used as each layer of the graph were the CIELAB color channels and the histogram of the image. 


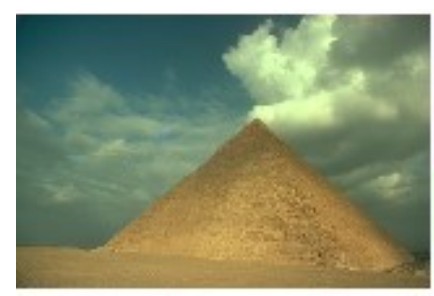

(a) Original

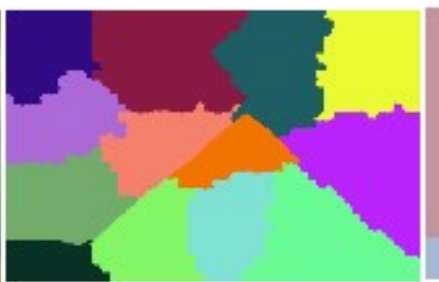

(b) Infomap

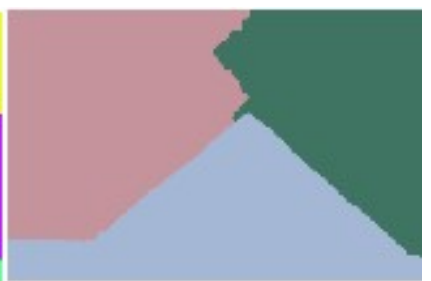

(c) Louvain

Figure 3. Original image (a), segmentation using Infomap (Multiplex Network)

(b) and segmentation using Louvain (Multiplex Network) (c)

As can be observed in Figure 3, the segmentations performed by the Informap method have several over segmentations and the changes are very sensitive, since the method based on the Louvain algorithm is more robust and does not present over segmentation and is also faster to execute. The Infomap algorithm takes about 0.5 seconds longer than the Louvain method, which consumes about 2 seconds, the time was not accounted for more precisely because it was not listed as an improvement factor proposed in this work. However, it is expected that the more communities the algorithm generates, the longer the processing time of the method, since the creation of more segments takes longer to process the output, the segmented image.

\subsection{EXPERImENT B: QUALitative ANALysis OF ImAge SEgmentation}

Considering the Berkeley image database and the segmentations presented in the human segmentation benchmark of this database, a comparison of the segmentations performed for a quick quality comparison is presented, as for each image there are several segmentations in this work, we present only the segmentation with the number of segments similar to the one returned in experiment $\mathrm{A}$.

The segmentation that most approached the segmentation based on the Infomap algorithm was the segmentation of the image in 11 communities. The segmentation that most approached the Louvain method was the segmentation of image with 3 communities.

As can be observed in Figures 4 and 5, where the green lines represent the segmented regions, although both segmentations did not obtain the same results, both segmentations are close to the benchmark used, especially the segmentation performed by the Louvain method. However, common subjectivity must be considered in segmentation tasks that vary widely by observer. But it should be added that rapid post-processing actions can still be implemented to minimize over segmentations in algorithms that provide outputs with these characteristics. 


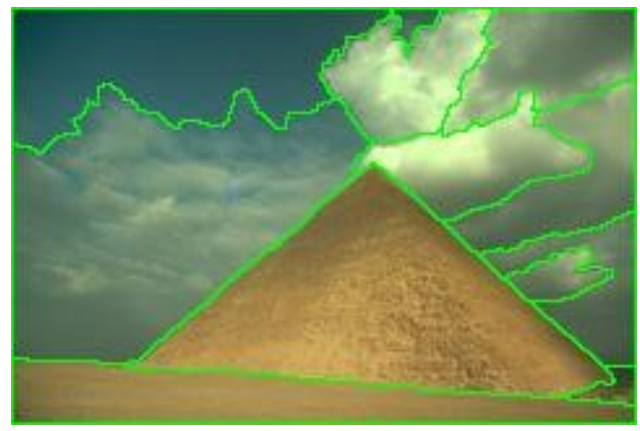

Figure 4. Human segmentation of image 299091.jpg of Berkeley Segmentation Dataset with 11 Segments

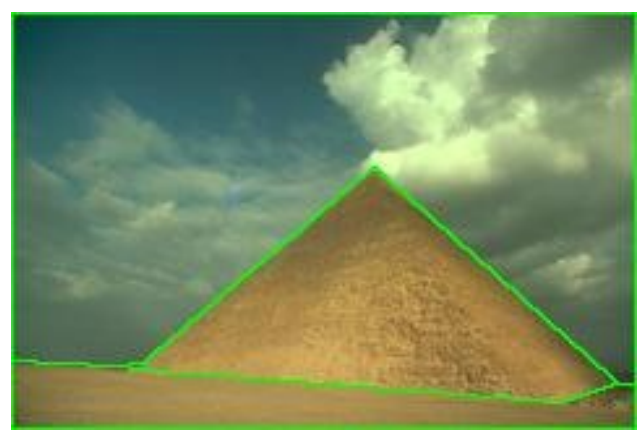

Figure 5. Human segmentation of image 299091.jpg of Berkeley Segmentation Dataset with 3 Segments

\section{Conclusions}

This work presents a useful method based on multiplex network and super pixels for the segmentation of images. Initial results provide quality segmentation. This method is also robust because it depends on specific parameters only in the pre-segmentation phase. It is interesting to note that such a method can act with several image features without the need of performing mathematical operations that can lead to the loss of feature information during the generation of graphs. However, issues related to the other properties that can be used deserve further investigation.

With the emergence of new generalizations in the area of multilayer networks it is interesting to review the algorithms used, since they can allow greater versatility and new adaptations in the method of segmentation of images. As future works, it is expected that multiplex networks will be replaced by multilayer networks with different super pixel sizes for each layer (property).

\section{REFERENCES}

[1] Gonzalez, Rafael C. and Woods, R. E. (2018). Digital Image Processing. 4th Edition. Prentice-Hall, Inc., Upper Saddle River, NJ, USA.

[2] Boykov, Y. and Funka-Lea, G. (2006). Graph cuts and efficient n-d image segmentation. Int. J. Comput Vision, 70(2):109-131. 
[3] Eriksson, A., Barr, O., and Astrom, K. (2006). Image segmentation using minimal graph cuts. In SSBA Symposium on Image Analysis.

[4] Vicente, S., Kolmogorov, V., and Rother, C. (2008). Graph cut based image segmentation with connectivity priors. In CVPR.

[5] Peng, B., Zhang, L., and Yang, J. (2010). Iterated graph cuts for image segmentation. In Proceedings of the 9th Asian Conference on Computer Vision - Volume Part II, ACCV'09, pp. 677-686, Berlin, Heidelberg. Springer-Verlag.

[6] Çigla, C. and Alatan, A. (2010). Efficient graph-based image segmentation via speeded-up turbo pixels. In Image Processing (ICIP), 2010 17th IEEE International Conference on, pp. 3013-3016.

[7] Hu, H., van Gennip, Y., Hunter, B., Bertozzi, A. L., and Porter, M. A. (2012). Multislice modularity optimization in community detection and image segmentation. In Vreeken, J., Ling, C., Zaki, M. J., Siebes, A., Yu, J. X., Goethals, B., Webb, G. I., e Wu, X., editors, ICDM Workshops, pp. 934-936. IEEE Computer Society.

[8] Lézoray, O. and Grady, L. J. (2012). Image Processing and Analysis with Graphs, Theory and Practice. CRC Press.

[9] Raghavan, U. N., Albert, R., and Kumara, S. (2007). Near linear time algorithm to detect community structures in large-scale networks. Physical Review E, 76(3):036106+.

[10] Wang, F., Wang, X., and Li, T. (2007). Efficient label propagation for interactive image segmentation. In Machine Learning and Applications, 2007. ICMLA 2007. Sixth International Conference on, pp. 136-141.

[11] Elqursh, A. and Elgammal, A. (2013). Online motion segmentation using dynamic label propagation. In Computer Vision (ICCV), 2013 IEEE International Conference on, pp. 2008-2015.

[12] Newman, M. E. J. and Girvan, M. (2004). Finding and evaluating community structure in networks. Phys. Rev. E, 69:026113.

[13] Abin, A. A., Mahdisoltani, F., and Beigy, H. (2014). Wisecode: wise image segmentation based on community detection. The Imaging Science Journal, 62(6):327-336.

[14] Browet, Arnaud \& Absil, Pierre-Antoine \& Van Dooren, Paul. (2011). Community Detection for Hierarchical Image Segmentation. 358-371.

[15] Mucha, P. J., Richardson, T., Macon, K., Porter, M. A., and Onnela, J.-P. (2010). Community structure in time-dependent, multiscale, and multiplex networks. Science, 328(5980):876-878.

[16] Kivelä, M., Arenas, A., Barthelemy, M., Gleeson, J. P., Moreno, Y., and Porter, M. A. (2014). Multilayer networks. Journal of Complex Networks.

[17] Linares, O. A., Botelho, G. M., Rodrigues, F. A., \& Neto, J. B. (2017). Segmentation of large images based on super-pixels and community detection in graphs. IET Image Processing, 11(12), 1219-1228.

[18] Domenico, M. De, Porter, M. A., Arenas, A. (2015). MuxViz: a tool for multilayer analysis and visualization of networks, Journal of Complex Networks, Volume 3, Issue 2, 1 June 2015, Pages 159 176. 


\section{Authors}

Ivo Socrates M. de Oliveira holds a degree in Information Systems from the Luterano University of Palmas (2007), a master's degree in Informatics from the University of Brasília (2013) and currently holds a doctorate in Computer Science and Computational Mathematics from the University of São Paulo, São Paulo. He is currently Professor at the Federal Institute of Tocantins. He has experience in the area of Computer Science, with emphasis on Image Processing, working mainly on the following themes: image segmentation and pattern recognition.

Oscar A. C. Linares holds a degree in Computer Science from Universidad Católica San Pablo, Peru (2008). Master's degree (2013) and PhD in Computer Science and Computational Mathematics from the University of São Paulo, Brazil. Did internship as scholarship researcher (2017) at the Institute for Data Analysis and Visualization (IDAV) at the University of California, Davis, United States. He has experience in the area of Computer Science, with emphasis on Image Processing, Complex Networks, Computer Graphics and Big Data.

Ary Henrique M. de Oliveira holds a degree in Information Systems from the Luterano University of Palmas (2002), a Master's Degree in Computer Science from the Fluminense Federal University (2006) and $\mathrm{PhD}$ in Systems and Computer Engineering from the Federal University of Rio de Janeiro (2015). He is currently Adjunct Professor II of the Federal University of Tocantins. Has experience in the area of Computer Science, with emphasis on Methodology and Computer Techniques. Acting mainly on the following topics: Cloud Computing, Reproducibility of Scientific Experiments, Electronic Science, Scientific Workflow, Scientific Workflow System and e-Science.

Glenda M. Botelho holds a degree in Computer Science from the Federal University of Goiás (2007), a master's degree (February 2011) and PhD (September 2014) in Computer Science from the Institute of Mathematical and Computer Sciences (ICMC) São Paulo (USP). She is currently Adjunct Professor II of the Computer Science course at the Federal University of Tocantins (UFT) and coordinator of the Nucleus of Applied Computing (NCA) -UFT. She works in the areas of image processing and artificial intelligence, focusing mainly on data/image analysis and machine learning.

João Batista Neto holds a degree in Computer Science from the Federal University of São Carlos (1988), a master's degree in Computer Science and Computational Mathematics from the University of São Paulo (1991) and PhD in Biomedical Engineering - Imperial College - University of London (1996). He is currently an MS-3 professor at the University of São Paulo, in the city of São Carlos. He has experience in Computer Science, with emphasis on Image Processing, working mainly on the following topics: segmentation, feature extraction, and pattern recognition.
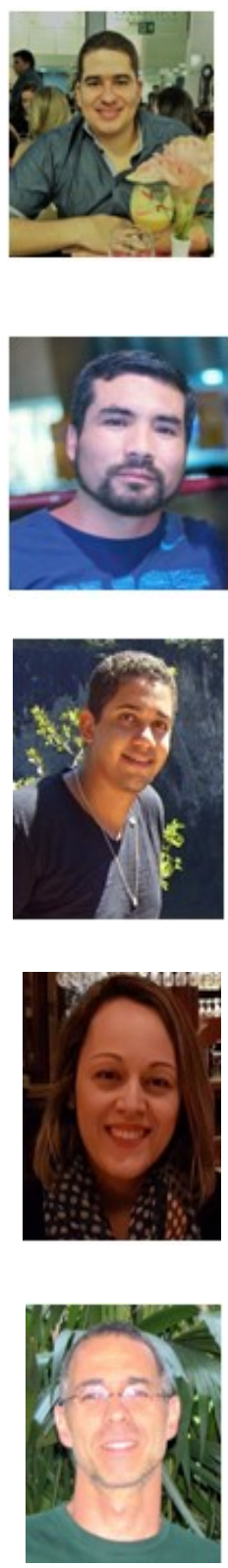